\title{
Histone acetylation and the role of histone deacetylases in normal cyclic endometrium
}

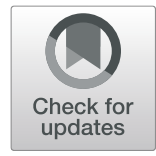

Palak Gujral', Vishakha Mahajan ${ }^{1,2}$, Abbey C. Lissaman ${ }^{1,2}$ and Anna P. Ponnampalam ${ }^{1,2,3^{*}}$

\begin{abstract}
Histone acetylation is a critical epigenetic modification that changes chromatin architecture and regulates gene expression by opening or closing the chromatin structure. It plays an essential role in cell cycle progression and differentiation. The human endometrium goes through cycles of regeneration, proliferation, differentiation, and degradation each month; each phase requiring strict epigenetic regulation for the proper functioning of the endometrium. Aberrant histone acetylation and alterations in levels of two acetylation modulators - histone acetylases (HATs) and histone deacetylases (HDACs) - have been associated with endometrial pathologies such as endometrial cancer, implantation failures, and endometriosis. Thus, histone acetylation is likely to have an essential role in the regulation of endometrial remodelling throughout the menstrual cycle.
\end{abstract}

Keywords: Histone acetylation, Histone deacetylation, Endometrium, Menstrual cycle

\section{Introduction}

The human endometrium is a dynamic tissue. Its fundamental function is to provide an immunoprivileged site for embryo implantation and a nurturing environment for fetal development [1]. It mainly consists of luminal epithelium, glandular epithelium, and endometrial stromal cells; which undergo regeneration, proliferation, differentiation, and degradation under the influence of steroid hormones estrogen and progesterone [1-4]. There are several genes involved in cyclic morphological and functional changes in the endometrium. These are upregulated or downregulated depending on the stage of the menstrual cycle, suggesting tight regulation of gene expression in the endometrium [5].

Epigenetic modification is a critical regulator of gene expression and determinator of cellular properties. Histone acetylation is a primary epigenetic change that makes genes accessible or inaccessible to transcriptional factors and thus influences gene expression. Histone acetylation,

\footnotetext{
* Correspondence: a.ponnampalam@auckland.ac.nz

'The Liggins Institute, The University of Auckland, Auckland, New Zealand

${ }^{2}$ Department of Obstetrics and Gynaecology, Faculty of Medical and Health

Sciences, The University of Auckland, Auckland, New Zealand

Full list of author information is available at the end of the article
}

in conjunction with other epigenetic modulators, has been associated with endometrial cyclic remodelling throughout the menstrual cycle; global histone acetylation levels have been seen to follow a cyclic pattern according to menstrual cycle stage in normal cyclic endometrium [6]. Histone acetylation is co-regulated by two sets of enzymes - histone acetyltransferases (HATs) and histone deacetylases (HDACs). Deregulation of HDACs and histone acetylation is often associated with endometrial pathologies such as cancer, endometriosis, and infertility [7-10]. However, there are very few studies explaining the role of histone acetylation and individual HDACs in endometrial stages and cell types. This review article summarizes the current literature on histone acetylation and the role of HDACs in normal cyclic endometrium and endometrial pathologies.

\section{Epigenetics}

Epigenetic alteration refers to the chemical or a physical modification that affects gene accessibility, thus regulating how genes are read and expressed, without changing the DNA sequence [11]. These modifications are both heritable and reversible. 
Some epigenetic modifications bring about lifechanging alterations in an organism, while others are just part of normal cellular functions [12]. Epigenetic modifications work in conjunction with genetic regulation to determine cellular properties and functions.

Genes are epigenetically regulated by DNA methylation, chromatin remodelling, and non-coding RNAs [13]. DNA methylation silences by directly adding a methyl group to cytosine in the DNA sequence, chromatin remodelling regulates expression by tightening and loosening chromatin structure, and non-coding RNAs attach to complementary sequences resulting in silencing of that gene. These epigenetic modifications work in coordination with each other to regulate gene expression [14]. DNA methyltransferases (DNMTs) regulate the DNA methylation in cells. Chromatin remodelling is primarily carried out through covalent histone modifications by enzymes such as HATs, HDACs, methyltransferases, and kinases [15]. Non-coding RNAs - including siRNAs, miRNAs, piRNAs, and long non-coding RNAs - epigenetically modify the genes by inducing transcriptional gene silencing [13].

Emerging research in epigenetics suggests that a range of environmental factors, lifestyle, early life stress, and trauma can influence the establishment and maintenance of epigenetic marks through generations (often known as epigenetic memory) $[11,16,17]$. Epigenetic reprogramming of induced pluripotent stem cells to develop novel treatments for diseases is an emerging area of research [18]. Epigenetic regulation is required for normal cellular functions; however, some epigenetic modifications can also lead to conditions such as cancer, developmental disorders, genomic imprinting disorders, $\mathrm{X}$-chromosome inactivation and endometriosis [19-21].

\section{Histone modifications}

Approximately $2 \mathrm{~m}$ of DNA is packaged inside a nucleus with the help of proteins called histones, forming a DNA-protein complex called chromatin. The fundamental unit of chromatin is called the nucleosome, which consists of two sets of the four core histones - H2A, H2B, H3 and H4 [22] (Fig. 1). Histone H1 links the nucleosomes together to form a chromosome [23]. This structure goes through many enzymatic modifications in-vivo, which gives rise to several genetic variabilities [24]. Histones have a protruding tail, which is subject to post-translational modifications (PTMs), such as acetylation, phosphorylation, lysine methylation, arginine

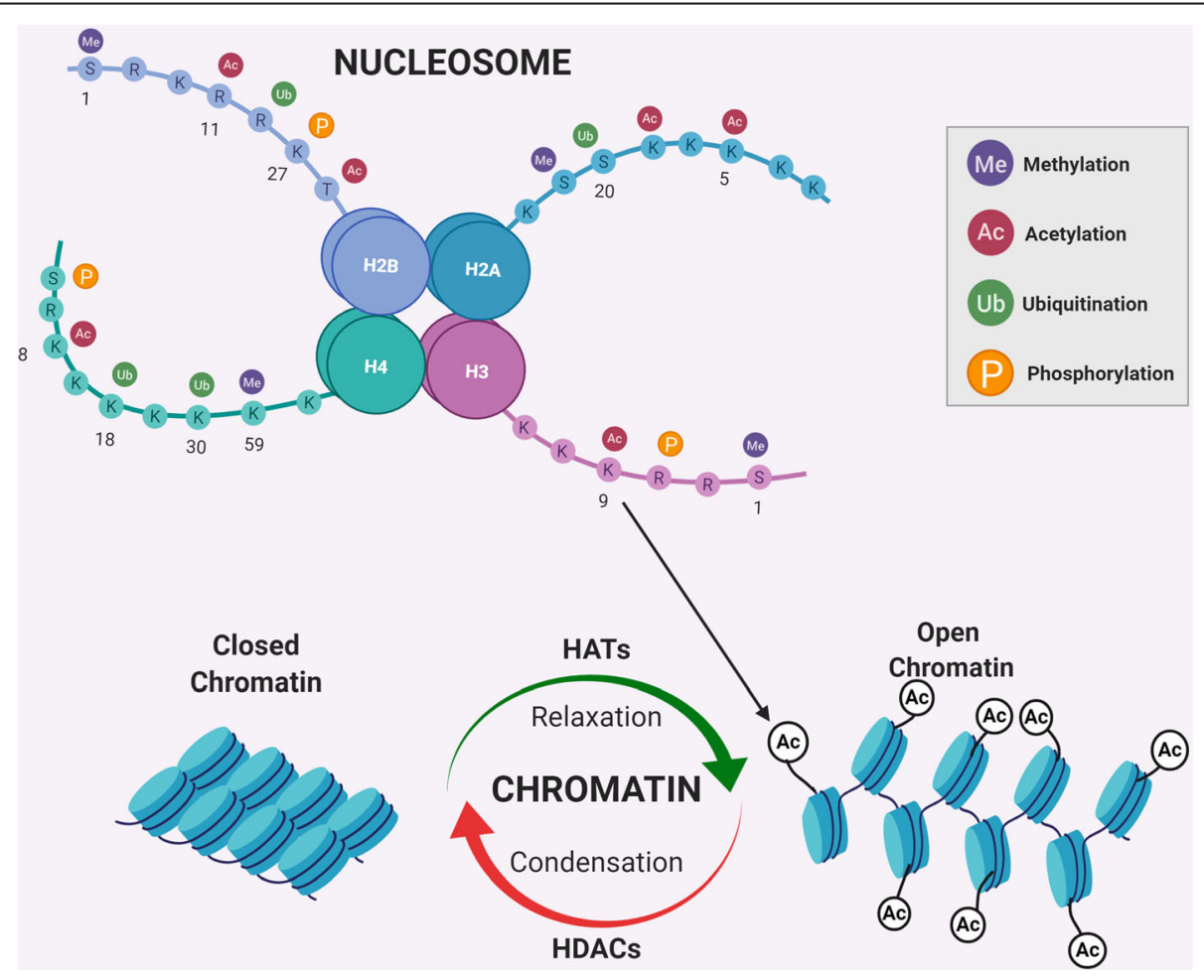

Fig. 1 Chromatin remodelling. Fundamental structure of chromatin called nucleosome consists of two sets of four histone proteins $\mathrm{H} 2 \mathrm{~A}, \mathrm{H} 2 \mathrm{~B}, \mathrm{H} 3$ and H4. Protruding histone tails undergo post translational modifications such as methylation, acetylation, ubiquitination and phosphorylation. The numbers indicate the positions of targeted lysine groups. Histone acetylation alters the conformation of chromatin structure in nucleus by relaxing the chromatin and allowing transcriptional activation. It is regulated by two sets of enzymes HATs and HDACs which add or remove acetyl group respectively from both histone and non-histone proteins, hence regulating gene transcription. (Created with BioRender.com) 
methylation, deamination, ADP ribosylation, proline isomerization, $\beta-\mathrm{N}$-acetylglucosamine, ubiquitylation, and sumoylation $[25,26]$. Histone modification regulates chromatin structure; facilitates remodelling; and affects DNA processes such as replication, repair, and recombination [25]. Most of these histone modifications are reversible and provide scope for developing novel therapeutic methods.

\section{Histone acetylation}

First identified in 1964 [27], histone acetylation involves the addition of an acetyl group to lysine residues in the protruding histone tails $[25,28]$. It is usually associated with transcriptional activation, and is modulated by two opposing groups of enzymes; histone acetyl transferases (HATs), which are responsible for adding acetyl groups; and histone deacetylases (HDACs), which remove them [25] (Fig. 1).

\section{Histone acetyltransferases}

HATs add an acetyl group to the $\varepsilon$-amino group of lysine using acetyl CoA as a cofactor, which neutralizes the positive charge on lysine, weakens the histone-DNA interaction, and makes genes accessible [25].

HATs are a diverse set of proteins. So far, about 30 HATs have been identified in humans. HATs are primarily classified into two classes based on their subcellular localization: Type A and Type B. Type A HATs are localized in the nucleus, while Type B is found in the cytoplasm. The Type A HATs function in transcriptionrelated histone acetylation in chromatin, while Type B HATs acetylate newly synthesized histones and influence the structure of the nucleosome $[29,30]$.

Type A HATs are further grouped into five families based on their catalytic domain [31, 32]. The Gcn5related $\mathrm{N}$-acetyltransferase (GNAT) family includes P300/CBP-associated factor (PCAF), Gnc5, and ELP3; the MYST family includes Tip60, monocytic leukemic zinc finger (MOZ), MOZ-related factor (MORF), human acetylase binding to ORC1 (HBO1), and human-malesabsent-on-the-first (HMOF) [32, 33]; the CBP/p300 family includes CBP and p300 [32, 34], and the transcription factor related family includes TAF1 and TIFIIIC90. Besides these, HATs also include many steroid receptor co-activators [32, 35, 36].

Deregulation of HATs is associated with cancer formation [37]. CBP/p300 is essential for the transition from G1 to S stage in the cell cycle and can function as either a tumor suppressor or an oncogene depending on its localization [38, 39]. Selective inhibition of p300 inhibits the cell cycle and induces apoptosis. It inhibits the response to DNA damage in melanoma cells [40]. MYST family HATs have various roles in stem cell function and development [33]. Because HATs are reversible regulators and are involved in cell cycle progression, they are being studied as targets for tumor growth management [41].

\section{Histone deacetylases}

HDACs are post-transcriptional modulators that remove acetyl groups from lysine residues of both histone and nonhistone proteins [42]. In humans, 18 HDACs have been identified and grouped into four classes based on their sequence homology to yeast [42, 43]: Class I (HDAC 1, 2, 3, and 8); class II (HDAC 4, 5, 6, 7, 9 and 10); class III (SIRT 1, 2, 3, 4, 5, 6 and 7) and class IV (HDAC 11). Class, I, II, and IV HDACs are $\mathrm{Zn}^{2+}$ dependent enzymes and have similar functional mechanisms whereas class III HDACs are NAD+ dependent. HDACs have relatively low substrate specificity, and one HDAC can act on multiple substrates or multiple HDACs can act on the same substrate [42]. Additionally, they work in the form of complexes with other HDACs and enzymes. These complications make it difficult to interpret the individual functions of HDACs [25]. There is strong evidence of cross-talk between HDACs and other epigenetic factors in the regulation of cancer tumorigenesis [44].

HDACs have proved to be dynamic enzymes that can modify a variety of proteins. The involvement of HDACs in regulating fundamental cellular functions such as proliferation, cell cycle, regeneration, apoptosis, and differentiation makes them an important target of study for disorders [43].

Class I HDACs are expressed in all tissues. HDAC 1 and 2 have been involved in cellular functions such as proliferation and apoptosis, while HDAC 3 is involved in the DNA damage response [43, 45]. HDACs 4, 5, 7, and 9 are associated with cell differentiation and development [45]. Sirtuins are mostly involved in cellular metabolism and DNA repair, while HDAC 11 is involved in regulating the expression of interleukins [46, 47]. Deregulation of HDACs is usually associated with cancer and tumorigenesis. Many HDAC inhibitors are now FDA approved anticancer drugs [48].

\section{The endometrium \\ The menstrual cycle}

Each month the endometrium goes through controlled cyclic changes, which are divided into three major phases: proliferative phase, secretory phase, and menstruation. This is called the menstrual cycle, and it occurs approximately 400 times in a lifetime [49].

The proliferative phase starts around day 5 of the menstrual cycle and ends around day 14 [50]. The proliferative phase of the endometrium runs parallel to the ovarian follicular cycle, and it is regulated by estrogen secreted from the developing ovarian follicle. The rising 
levels of estrogen help restore the funtionalis layer of the endometrium [51], leading to the thickening of the endometrium, growth of endometrial glands, and emergence of spiral arteries [52]. The global acetylation levels of H2AK5, H3K9, and H4K8 in human cyclic endometrium have been seen to be elevated in the early proliferative phase [6]. This may be associated with the requirement of transcriptional activation of regenerative and proliferative pathways during the early proliferative phase. The acetylation levels decline in later stages until ovulation, suggesting a switch from proliferative to secretory functions [6] (Fig. 2).

Post ovulation, around day 14 of the menstrual cycle, there is an increase in progesterone levels produced by the corpus luteum, which gives rise to secretory changes in the endometrium [53]. The first half of the secretory phase involves glandular changes; stromal changes are more evident during the second half [53]. Spiral arteries and glands become tortuous during this phase [2]. The endometrial cells go through morphological and functional changes to differentiate and prepare for implantation, a process called decidualization. The human endometrium is decidualized in each menstrual cycle [54]. A significant increase in acetylation levels of H4K8 has been seen post ovulation, and a similar trend has also been observed in H3K9 and H4K14/18 (Fig. 2). This correlates with the requirement for secretory modifications like cellular differentiation, angiogenesis, and decidualization [6].
In the absence of a fertilized embryo, the fall in progesterone levels triggers the breakdown of the decidualized endometrial lining. This process is called menstruation [2], and correlates with the decline in histone acetylation levels seen in the late secretory phase [6] (Fig. 2).

Interestingly, other estrogen and progesterone responsive tissues such as breast, fallopian tubes, and vagina do not shed in response to the decline in hormonal levels [2]. After menstruation, the endometrial lining starts to regenerate and repair to prepare for the next cycle.

These perfectly timed events of regeneration, proliferation, differentiation, and degradation require strict epigenetic and genetic regulation of endometrial remodelling. Many endometrial pathologies are associated with the deregulation of epigenetic control. Understanding the epigenetic regulation of the endometrial lining is also essential in order to improve artificial reproductive technologies by identifying the receptive endometrium and modulating the endometrial receptivity state [55].

\section{Regeneration}

After each menstrual cycle, the endometrial functionalis layer regenerates from the stratum basalis. It grows from an initial thickness of $0.5-1.0 \mathrm{~mm}$ to up to $7.0-8.0 \mathrm{~mm}$ in the secretory phase [53]. It not only regenerates during the menstrual cycle, but also following parturition;

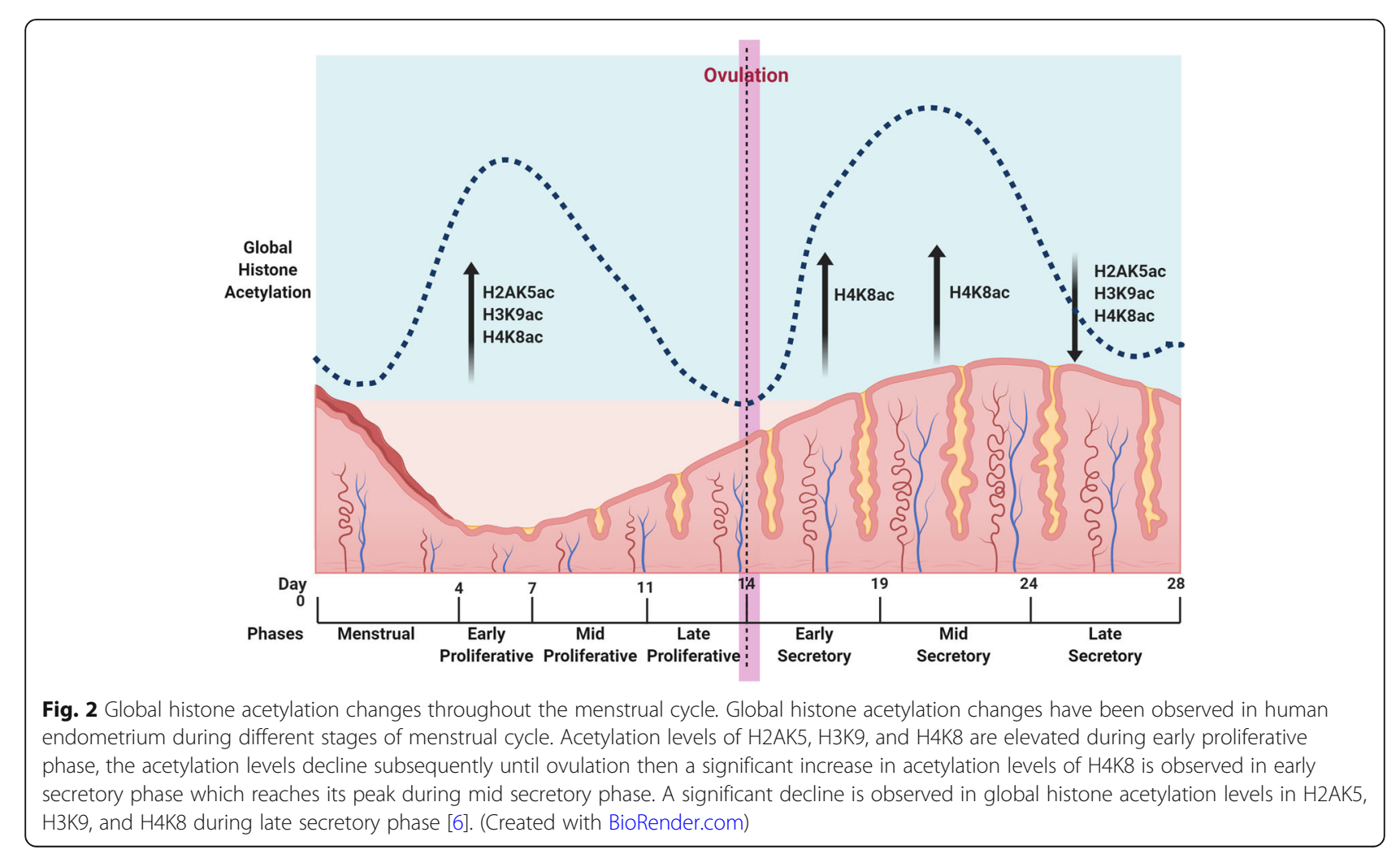


almost complete resection; and in postmenopausal women taking hormone replacement therapy [56, 57]. Endometrial re-epithelialization in humans occurs rapidly and is scar-free [58]. For several years, epigenetic regulation has been linked to regeneration processes [59]. HDAC inhibitors or histone acetylating agents are capable of inducing scar-free wound healing by stimulating cytokines or growth factors, which are crucial for rapid re-epithelialization [60].

The remarkable regenerative capacity of the endometrium is due to the presence of adult progenitor stem cells [61]. These cells are found throughout the body and play a crucial role in the regeneration and repair of damaged tissues and the maintenance of organs. Epithelial progenitor cells and mesenchymal stem/stromal cells play an essential role in the regeneration and repair of endometrial epithelium and stroma [56]. A small population of mesenchymal stem cells (MSC) - a subpopulation of adult progenitor stem cells - can be found in the endometrium and are called endometrial MSCs (eMSC) $[62,63]$. They are highly regenerative stem cells that have similar properties to bone marrow MSCs [56]. Menstrual blood also contains clonogenic, multipotent MSCs, which have a broad differentiation capacity [64].

Over the past decade, several studies have shown that epigenetics are a crucial regulator of stem cell functioning [65]. Histone modification changes chromatin architecture and regulates gene expression. Since histone acetylation is an essential post-transcription modulator, it is a basic requirement in stem cell functioning and works in coordination with DNA methylation activity. The fate determination of MSCs is controlled by a complex network of transcription factors and histonemodifying enzymes [66]. HDAC 1 silencing improves the efficiency of human umbilical cord MSCs in mouse models with traumatic brain injury [67]; while HDAC 6 deficiency, causing acetylation of p53 K120, can induce apoptosis in MSCs [68]. Global H3K9Ac level decreases, and $\mathrm{H} 3 \mathrm{~K} 9 \mathrm{Me} 2$ increases during osteogenic differentiation of MSCs [69]. These studies indicate the involvement of histone acetylation in the regulation of MSC function.

The endometrium has become a popular source of stem cells for reprogramming into induced pluripotent stem cells (iPSCs) [56]. The MSCs derived from the endometrium and umbilical cord are being used as iPSCs in regenerative medicine, exploiting the epigenetic regulation of these cells [70, 71]. HDAC inhibitors such as valproic acid (VPA), trichostatin A (TSA), suberoylanilide hydroxamic acid (SAHA), and butyrate have been known to enhance reprogramming in iPSCs [72].

Aberrant epigenetic regulation in eMSCs has been associated with endometriosis pathogenesis [73]. eMSCs are being targeted for the development of novel therapies for the treatment of endometriosis via epigenetic reprogramming [74].

Upregulation of the global acetylation levels of $\mathrm{H} 2 \mathrm{AK} 5, \mathrm{H} 3 \mathrm{~K} 9$, and $\mathrm{H} 4 \mathrm{~K} 8$ during the early proliferative phase in the adult endometrium correlates with transcription activation of several regenerative pathways [6]. Although changes in histone acetylation levels and HDACs in normal to diseased conditions have been discussed, and known evidence of epigenetic involvement in endometrial regeneration is available, the target genes and level of HDAC expression throughout the menstrual cycle have not yet been identified.

Histone acetylation and DNA-methylation work in a coordinated manner, decide stem cell fate and influence cancer pathogenesis [75].

\section{Proliferation}

After endometrium regeneration, estrogen levels from the developing follicle stimulate the proliferation of stromal and epithelial cells during the proliferative phase of the menstrual cycle [56, 76]. Estrogen and progesterone surface receptors increase in number, and the rapid formation of new blood vessels, called angiogenesis, occurs to support the nutritional requirements of the growing tissue [77].

Cancer studies provide us with great insight into the epigenetic regulation of cell proliferation. HDACs can act as both suppressors and inducers of cell proliferation, depending on the gene they regulate. Inhibition of HDAC 1 reduces cell proliferation, and inhibition of HDAC 3 is associated with decreased migration of ovarian cancer cells [78].

Class I HDACs $[1-3,8]$ are critical for modulating cell survival and proliferation, and HDACs 1,2 , and 3 play an important role in steroid hormone-dependent gene expression in the human endometrium $[9,10]$. A previous study on human cyclic endometrial tissues has demonstrated that the global acetylation levels of H2AK5, $\mathrm{H} 3 \mathrm{~K} 9$, and $\mathrm{H} 4 \mathrm{~K} 8$ are elevated in the early proliferative phase, and are associated with the transcriptional activation of proliferative and regenerative pathways [6]. HDAC expression is upregulated in most endometrial cancers compared to normal cyclic endometrium [79]. A study on endometrial stromal sarcoma (ESS) showed that expression of HDACs 1 and 2 is higher in ESS compared to non-neoplastic stem cells, with HDAC 2 expression being slightly higher than HDAC 1 . Studies also show that inhibition of HDAC 2 can lead to cell differentiation and inhibition of proliferation [80, 81]. HDAC inhibitors can induce expression of p21 and p27 (endogenous cyclin-dependent kinase inhibitors), which cause cell cycle arrest and inhibit proliferation [82]. Several studies have used HDAC inhibitors as anti-cancer agents to normalize cell proliferation in endometrial 
carcinomas [10, 79, 83-85]. SAHA, m-carboxycinnamic acid Bishydroxamide (CBHA), Scriptaid, Oxamflatin, VPA, Sodium Butyrate, M344, Apicidine, Psammaplin A (PsA) and MS-275 are HDAC inhibitors that have been successfully studied in endometrial cancers and are found to induce cell cycle arrest and regulate proliferation [79].

Studies using HDAC inhibitors VPA and SAHA have also shown the association between HDACs and angiogenesis in vitro. The study showed that treatment with HDAC inhibitors VPA and SAHA, in combination with vascular endothelial growth factor (VEGF), increased endothelial cell sprouting [86], suggesting an association between histone acetylation and angiogenesis. Because angiogenesis is crucial for endometrial repair and reepithelialization [87], the role of histone acetylation in angiogenesis is critical for menstrual cycle regulation.

\section{Decidualization and implantation}

The rise in progesterone level post ovulation gives rise to morphological and functional changes in the human endometrium called decidualization. These changes include differentiation of fibroblastoid endometrial stromal cells (ESC) into decidual cells, the presence of decidual white blood cells, and vascular modifications in maternal arteries [54, 88]. Decidualization initiates a cascade of events that allow the embryo to attach to the endometrium and coordinates the trophoblast invasion [89]. Invasion of trophoblast into endometrial decidua requires tissue remodelling enzymes [90]. Matrix metalloproteinases (MMPs) are a group of enzymes required for tissue remodelling and degrading extracellular matrix (ECM) [91, 92]. During implantation and trophoblast invasion, MMP-2, and MMP-3 are expressed and secreted by human endometrium. In contrast, the decidual stromal cells express tissue inhibitors of MMPs (TIMPs) that limit the degradation of ECM by MMPs, thus hindering trophoblast invasion. A balance between MMPs and TIMPs in the endometrium is required for successful implantation of the blastocyst [91, 93, 94].

Decidualization, being a complicated process, requires dramatic gene expression changes in ESCs [88]. These changes are associated with histone modifications, and several studies from the past two decades have shown a correlation between decidualization and histone modifications. In the previous study by Munro et al., on human endometrial tissue biopsies, a statistically significant increase in H4K8 expression was seen, and H3K9 and H4K14/18 followed a similar trend [6]. These findings correlate well with another study on ESCs cultured with estrogen and progesterone, which showed a significant increase in the acetylation of H4K8 and H3K9/14 [95]. In a genome-wide analysis of histone modifications in human ESCs, H3K27ac and H3K4me3 levels were high during decidualization. Genes with increased H3K27ac and H3K4me3 levels were found to be involved in insulin signaling pathways, crucial for the decidualization process [88].

Trichostatin A (TSA) is an inhibitor of HDAC and is involved in the inhibition of HDACs class I, II, and IV; TSA however, does not inhibit class III HDACs (Sirtuins) [96]. A comparative study between cultured ESCs and glandular cells isolated from human endometrium showed that the addition of TSA enhances the upregulation of decidualization markers such as insulin-like growth factor binding protein-1 (IGFBP-1) and prolactin, as regulated by $17 \beta$-estradiol (E2) plus progesterone (P4) [95]. This study showed the involvement of histone acetylation and the connection of HDACs to the decidualization of the endometrium, suggesting that TSA acts as an enhancer of the decidualization process. In contrast, a decade later, another study showed that treatment of human ESCs with TSA had an inhibitory effect on trophoblast invasion. The ESCs treated with TSA showed increased TIMP-1 and TIMP-3 expression, while expression of MMPs was decreased. This study associated histone acetylation with the disruption of trophoblast invasion and suggested HDACs are critical for implantation [91]. A recently published study on mice demonstrated that loss of HDAC 3 in the uterus of mice leads to implantation failures. Interestingly, HDAC 3 is usually the least frequently expressed HDAC in the endometrial tissue $[7,10]$. In the study, HDAC 3 was shown to be involved in transcription activation of COL1A1 and COL1A2 (collagenase) genes in humans and colla1 and colla2 genes in mice [7]. Collagenases are involved in endometrial remodelling and trophoblast invasion during implantation [91]. The cumulative findings of these studies suggest that HDAC 3 could be crucial for the implantation process, but in a limited capacity. A tightly controlled relationship between HATs and HDACs is essential for proper decidualization and successful implantation.

\section{Histone acetylation and endometrial pathologies}

The highly timed molecular events discussed above, if not appropriately regulated, lead to a spectrum of endometrial pathologies such as endometriosis, endometrial cancers, abnormal uterine bleeding, and infertility.

Studies have found the involvement of histone acetylation, in conjunction with other epigenetic modulators, in several human malignancies. High expression of class I HDACs in some tumors has been linked to unfavorable prognosis, with the exception of breast cancer in which it has a favorable prognosis. On the other hand, the upregulation of class II HDACs is associated with a favorable prognosis in human tumors [97]. One of the earliest studies on endometrial stromal sarcoma (ESS) showed 
that HDAC 2 expression is upregulated in ESS compared to the non-neoplastic endometrial stroma [80]. They also showed that HDAC 1 expression is generally lower than HDAC 2, and inhibition of HDAC 2 using valproate affects cell differentiation by increasing the expression of cell cycle regulators [80]. Later, a study on endometrial adenocarcinomas showed a decrease or complete loss of epithelial HDAC 1 protein expression compared to normal endometrium [10]. Alternatively, a study on endometrial carcinomas (EC) showed that HDAC 1, 2 and 3 expression levels are increased in ECs compared to healthy endometrial tissues [85]. Although this study did not account for the cycle stages of the endometrium in analysis, it still showed similarity with earlier studies, in that HDAC 2 is the most expressed in the endometrium, while HDAC 3 is the least expressed $[10,85]$. An immunohistochemical study on endometrial carcinoma showed that expression of HDAC 1, HDAC 2, and Ki-67 (a cellular marker of proliferation) are higher in endometrial carcinomas than in normal endometrium. The treatment of endometrial carcinoma cell lines with HDAC inhibitors TSA and apicidin reduces cell proliferation and increases p21 expression. Apicidin also reduces cyclin D1 and CDK4 expression [81, 98]. HDAC inhibitors Vorinostat, Romidepsin (FK228), and LBH589 help induce cell cycle arrest in endometrial carcinomas [98-100]. Progesterone induces differentiation in endometrial cells and inhibits proliferation through the action of progesterone receptors (PR). Therefore, the loss of PR also leads to endometrial carcinomas. Treatment of ECC-1 and Ishikawa $\mathrm{H}$ cells with the HDAC inhibitor LBH589 has been shown to increase $P R$ expression, which in turn regulates cell differentiation and induces cell cycle arrest [101, 102]. While most HDACs are upregulated in ECs, recent studies have shown that SIRT 6 induces apoptosis and inhibits proliferation in endometrial cancer cells by repressing survivin [103]. Expression levels of SIRT 1, SIRT 2, SIRT 4, and SIRT 5 are seen to be downregulated, while SIRT 7 is significantly upregulated in ECs compared to nonneoplastic cells. Interestingly, this study showed no significant difference in SIRT 6 and SIRT 3 levels between ECs and non-neoplastic cells [104] (Fig. 3).

Histone acetylation and aberrant levels of HDACs are also associated with endometriosis. HDAC 1 expression is seen to be significantly elevated during endometriosis compared to normal endometrium, and the level also correlates with low acetylation levels of $\mathrm{H} 3$ and $\mathrm{H} 4$ [9, 106]. It was seen again in a later study that HDAC 1 and 2 expression levels were high in endometriotic stromal cells compared to healthy endometrial stromal cells. Differential expression of HDAC 1 and 2 was observed

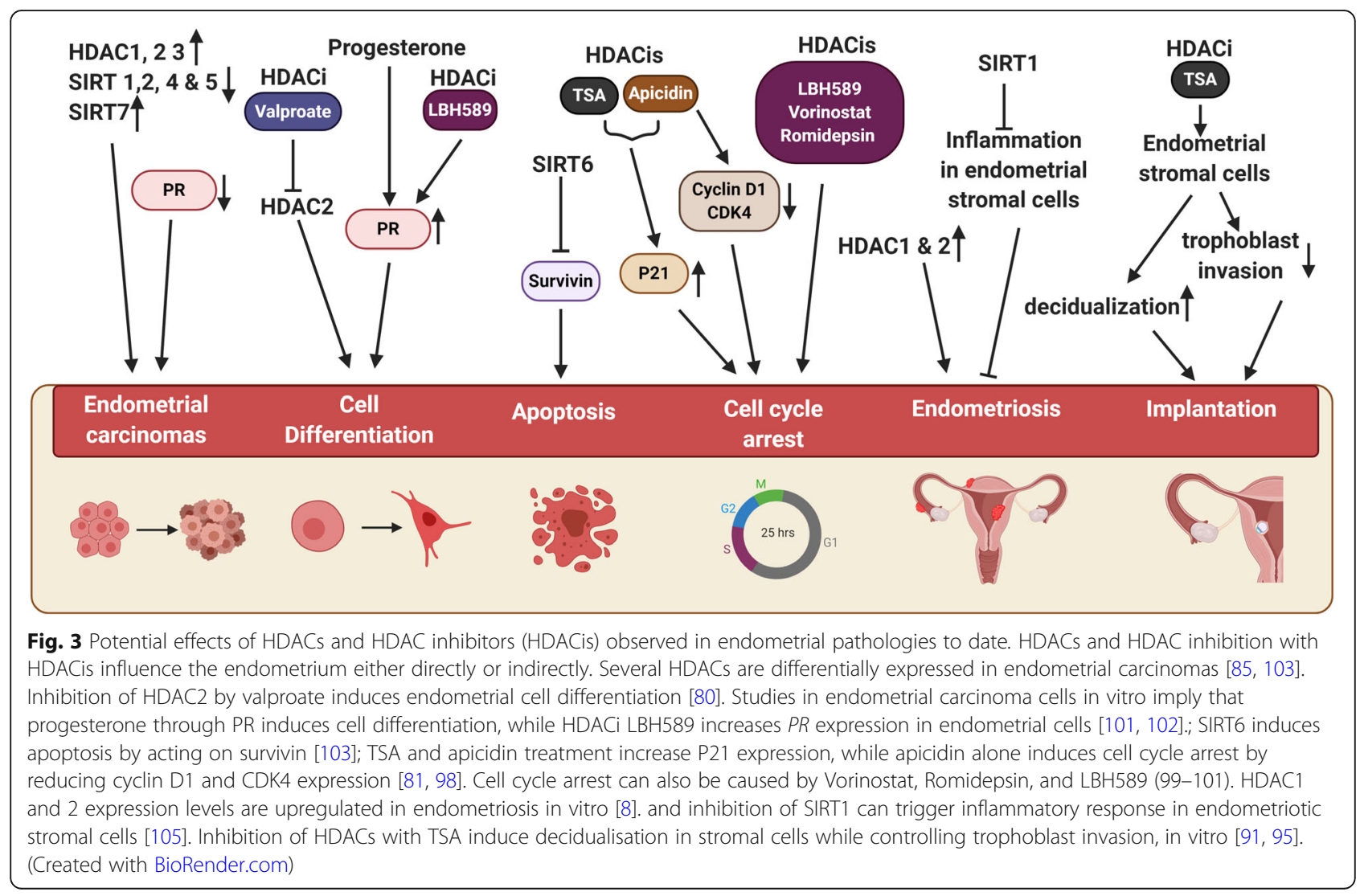


based on lesion type and localization in endometrioid cells [8]. A comparative study between ectopic and eutopic endometrial samples taken from women who have endometriosis showed that $H D A C 1$ gene expression was high in ectopic tissues while $H D A C 2$ expression levels were high in eutopic tissue samples. Expression of HATs P300 and CREBBP did not change significantly when endometriosis tissue samples were compared to standard samples, while PCAF expression was high in the ectopic tissue [107]. It should be noted that this study used total tissue samples with minimal sample size, and the total sample contained varied cell populations from various endometrial cycle stages. The antiinflammatory effects of SIRT1 have also been investigated in endometriosis. This study compared the action of SIRT1 and its activator resveratrol in endometriotic stromal cells and healthy endometrial stromal cells. They found that the activation of SIRT1 suppresses inflammatory responses in endometriotic stromal cells, while inhibition of SIRT1 can trigger inflammatory responses. Suggesting its crucial role in maintaining healthy endometrial stromal cells [105] (Fig. 3).

HDACs also play a crucial role in implantation and decidualization. Loss of $H D A C 3$ is linked to decidualization defects and implantation failure in mice [7]. HDAC inhibitor TSA in endometrial stromal cells negatively regulates trophoblast invasion and facilitates decidualization, influencing embryo implantation [91, 95] (Fig. 3).

Since most of the studies on endometriosis and other endometrial pathologies compare healthy and diseased tissue samples, it is hard to tell if the epigenetic aberrations are the cause or effect of various endometrial pathologies.

\section{Conclusions}

Histone acetylation is a fundamental regulator of chromatin structure and gene expression. Many studies on endometrial tissues and cell lines have shown the involvement of aberrant levels of histone acetylation and HDACs in endometrial pathologies, especially endometrial carcinomas and endometriosis. The majority of HDACs studied seem to be elevated in endometrial carcinomas compared to the normal endometrium $[8,85]$. High levels of HDACs are also associated with endometriosis in many women. Furthermore, evidence suggests that histone acetylation and HDACs are involved in various endometrial pathologies [7-9, 79, 83]. However, most of these studies have not given any regard to the cyclic nature of the endometrium. HDACs are being studied as potential therapeutic targets for endometrial carcinomas and endometriosis; their effect reversed with HDAC inhibitors [79, 83]. Global histone acetylation levels of H2AK5, H3K9, and H4K8 are found to be elevated in the early proliferative phase of the endometrium, then decline until ovulation. The acetylation levels in $\mathrm{H} 4 \mathrm{~K} 8$ rise significantly post ovulation, and a similar trend is observed in $\mathrm{H} 3 \mathrm{~K} 9$ as well as H4K14 and 18 (Fig. 2). This study suggests that histone acetylation trends seem to follow a cyclic pattern in coordination with menstrual cycle events that require transcriptional activation and silencing [6]. HDACs function in conjunction with other epigenetic modulators, regulating molecular modifications in the endometrium [107]. Studying the general levels of HDACs and histone acetylation in normal cyclic endometrium will give us insight into individual functions and targets of individual HDACs. Because histone acetylation is a reversible epigenetic mark, studying its regulation will eventually help us to develop targeted therapies to improve the menstrual health of women.

\section{Abbreviations}

DNMT: DNA methyltransferase; EC: Endometrial carcinomas;

ECM: Extracellular matrix; eMSC: Endometrial mesenchymal stem cells; ESC: Endometrial stromal cell; ESS: Endometrial stromal sarcoma; FDA: U.S.

Food and Drug Administration; GNAT: Gcn5-related N-acetyltransferase; HAT: Histone acetyltransferase; HBO1: Human acetylase binding to ORC1; HDAC: Histone deacetylase; HDACi: Histone deacetylase inhibitor; HMOF: Human-males-absent-on-the-first; iPSC: Indiced pluripotent stem cells; MMP: Matrix metalloproteinases; MORF: MOZ related factor; MOZ: Monocytic leukemic zinc finger; MSC: Mesenchymal stem cells; PCAF: P300/CBPassociated factor; PR: Progesterone receptor; PTM: Post-translational modification; SAHA: Suberoylanilide hydroxamic acid; TIMPS: Tissue inhibitors of MMPs; TSA: Trichostatin A; VEGF: Vascular endothelial growth factor; VPA: Valporic acid

\section{Authors' contributions}

PG: Overall drafting of the review article, writing and desiging the Figs. VM: Helped in deciding the flow of the article, editing and proof reading. AL: Editing and proof reading of the article. AP: Overall supervision, conception and editing the article. The author(s) read and approved the final manuscript.

Funding

This work was supported by Health Research Council of New Zealand (17/632) and Auckland Medical Research Foundation (1116005).

Availability of data and materials Not applicable.

Ethics approval and consent to participate Not applicable.

Consent for publication

Not applicable.

\section{Competing interests}

The authors declare that they have no competing interests.

\section{Author details}

TThe Liggins Institute, The University of Auckland, Auckland, New Zealand. ${ }^{2}$ Department of Obstetrics and Gynaecology, Faculty of Medical and Health Sciences, The University of Auckland, Auckland, New Zealand. ${ }^{3}$ Department of Physiology, Faculty of Medical and Health Sciences, The University of Auckland, Private Bag 92019, Auckland 1142, New Zealand. 
Received: 8 March 2020 Accepted: 29 July 2020

Published online: 13 August 2020

\section{References}

1. Fiac J, Jiménez-Ayala M, Jiménez-Ayala Portillo B. Cytopathology of the glandular lesions of the female genital tract. In: Orell SR, editor. Monographs in clinical cytology. Vol. 20. Basel (Suiza): Karger Eds; 2011. ISBN 978-3-80559464-6. Progresos de Obstetricia y Ginecología. 2011.

2. Lessey BA, Young SL. Chapter 9 - structure, function, and evaluation of the female reproductive tract. In: Strauss JF, Barbieri RL, editors. Yen and Jaffe's reproductive endocrinology (8th edition). Philadelphia: Content Repository Only! 2019. p. 206-47.e13.

3. Jiménez-Ayala M, Jiménez-Ayala Portillo B. Cytology of the Normal endometrium - cycling and postmenopausal. In: Endometrial Adenocarcinoma: Prevention and Early Diagnosis. Basel, S. Karger AG. 2008 p. 32-9.

4. Kimball FA. The endometrium. Dordrecht: Springer Netherlands 1980; 1980

5. Yamagata Y, Asada H, Tamura I, Lee L, Maekawa R, Taniguchi K, et al. DNA methyltransferase expression in the human endometrium: down-regulation by progesterone and estrogen. Hum Reprod. 2009;24(5):1126-32.

6. Munro SK, Farquhar CM, Mitchell MD, Ponnampalam AP. Epigenetic regulation of endometrium during the menstrual cycle. Mol Hum Reprod. 2010;16(5):297-310.

7. Kim TH, Yoo J-Y, Choi K-C, Shin J-H, Leach RE, Fazleabas AT, et al. Loss of HDAC3 results in nonreceptive endometrium and female infertility. Sci Transl Med. 2019;11(474):eaaf7533.

8. Colón-Díaz M, Báez-Vega P, García M, Ruiz A, Monteiro JB, Fourquet J, et al. HDAC1 and HDAC2 are differentially expressed in endometriosis. Reprod Sci. 2012;19(5):483-92.

9. Samartzis EP, Noske A, Samartzis N, Fink D, Imesch P. The expression of histone deacetylase 1, but not other class I histone deacetylases, is significantly increased in endometriosis. Reprod Sci. 2013;20(12):1416-22

10. Krusche CA, Vloet AJ, Classen-Linke I, von Rango U, Beier HM, Alfer J. Class I histone deacetylase expression in the human cyclic endometrium and endometrial adenocarcinomas. Hum Reprod. 2007;22(11):2956-66.

11. Krippner S, Barrett D. Transgenerational trauma: the role of epigenetics. J Mind Behavior. 2019:40(1):53-62.

12. Jaenisch R, Bird A. Epigenetic regulation of gene expression: how the genome integrates intrinsic and environmental signals. Nat Genet. 2003; 33(3):245-54.

13. Wei JW, Huang K, Yang C, Kang CS. Non-coding RNAs as regulators in epigenetics (review). Oncol Rep. 2017;37(1):3-9.

14. Geiman TM, Robertson KD. Chromatin remodeling, histone modifications, and DNA methylation? How does it all fit together? Journal of cellular biochemistry. 2002;87(2):117-25.

15. Teif VB, Rippe K. Predicting nucleosome positions on the DNA: combining intrinsic sequence preferences and remodeler activities. Nucleic Acids Res. 2009;37(17):5641-55.

16. Cavalli $G$, Heard $E$. Advances in epigenetics link genetics to the environment and disease. Nature. 2019;571(7766):489-99.

17. Nilsson E, Ben Maamar M, Skinner MK. Chapter 2 - definition of epigenetic transgenerational inheritance and biological impacts. In: Tollefsbol TO, editor. Transgenerational epigenetics (2nd edition). Vol. 13. Oxford: Academic; 2019. p. 11-16.

18. Karagiannis P. Clinical Potential of Induced Pluripotent Stem Cells. In H. Inoue \& Y. Nakamura, editors. Medical Applications of iPS Cells: Innovation in Medical Sciences. Singapore. Singapore: Springer; 2019. p. 3-12.

19. Ayers D, Boughanem H, Macías-González M. Epigenetic influences in the obesity/colorectal cancer Axis: a novel Theragnostic avenue. J Oncol. 2019; 2019:7406078

20. Yeshurun S, Hannan AJ. Transgenerational epigenetic influences of paternal environmental exposures on brain function and predisposition to psychiatric disorders. Mol Psychiatry. 2019;24(4):536.

21. Monk D, Mackay DJG, Eggermann T, Maher ER, Riccio A. Genomic imprinting disorders: lessons on how genome, epigenome and environment interact. Nat Rev Genet. 2019;20(4):235-48.

22. Pathak R, Singh P, Ananthakrishnan S, Adamczyk S, Schimmel O, Govind CK Acetylation-dependent recruitment of the FACT complex and its role in regulating pol II occupancy genome-wide in saccharomyces cerevisiae. Genetics. 2018;209(3):743-56.
23. Annunziato A. DNA packaging: nucleosomes and chromatin. Nature Educ. 2008;1(1):26.

24. Turner BM. Histone acetylation and an epigenetic code. Bioessays. 2000; 22(9):836-45.

25. Bannister AJ, Kouzarides T. Regulation of chromatin by histone modifications. Cell Res. 2011:21:381.

26. Keck KM, Pemberton LF. Histone chaperones link histone nuclear import and chromatin assembly. Biochim Biophys Acta. 2012;1819(3-4):277-89.

27. Allfrey $V$, Faulkner R, Mirsky A. Acetylation and methylation of histones and their possible role in the regulation of RNA synthesis. Proc Natl Acad Sci. 1964;51(5):786-94.

28. Ellenbroek B, Youn J. Chapter 5 - environment challenges and the brain. In: Ellenbroek B, Youn J, editors. Gene-environment interactions in psychiatry. San Diego: Academic; 2016. p. 107-39.

29. Richman R, Chicoine LG, Collini MP, Cook RG, Allis CD. Micronuclei and the cytoplasm of growing Tetrahymena contain a histone acetylase activity which is highly specific for free histone H4. J Cell Biol. 1988;106(4):1017-26.

30. Roth SY, Denu JM, Allis CD. Histone Acetyltransferases. 2001;70(1):81-120.

31. Lee KK, Workman JL. Histone acetyltransferase complexes: one size doesn't fit all. Nat Rev Mol Cell Biol. 2007;8(4):284-95.

32. Sun XJ, Man N, Tan Y, Nimer SD, Wang L. The role of histone acetyltransferases in Normal and malignant hematopoiesis. Front Oncol. 2015:5:108

33. Voss AK, Thomas T. MYST family histone acetyltransferases take center stage in stem cells and development. Bioessays. 2009;31(10):1050-61.

34. Bowers EM, Yan G, Mukherjee C, Orry A, Wang L, Holbert MA, et al. Virtual ligand screening of the p300/CBP histone acetyltransferase: identification of a selective small molecule inhibitor. Chem Biol. 2010; 17(5):471-82.

35. Spencer TE, Jenster G, Burcin MM, Allis CD, Zhou J, Mizzen CA, et al. Steroid receptor coactivator-1 is a histone acetyltransferase. Nature. 1997:389(6647):194

36. Chen H, Lin RJ, Schiltz RL, Chakravarti D, Nash A, Nagy L, et al. Nuclear receptor coactivator ACTR is a novel histone acetyltransferase and forms a multimeric activation complex with P/CAF and CBP/p300. Cell. 1997;90(3): 569-80.

37. Gajer JM, Furdas SD, Gründer A, Gothwal M, Heinicke U, Keller K, et al. Histone acetyltransferase inhibitors block neuroblastoma cell growth in vivo. Oncogenesis. 2015;4(2):e137-e.

38. Dekker FJ, Haisma HJ. Histone acetyl transferases as emerging drug targets. Drug Discov Today. 2009;14(19):942-8.

39. Ait-Si-Ali S, Polesskaya A, Filleur S, Ferreira R, Duquet A, Robin P, et al. CBP/ p300 histone acetyl-transferase activity is important for the G1/S transition. Oncogenesis. 2000;19(20):2430.

40. Yan G, Eller MS, Elm C, Larocca CA, Ryu B, Panova IP, et al. Selective inhibition of p300 HAT blocks cell cycle progression, induces cellular senescence, and inhibits the DNA damage response in melanoma cells. J Investig Dermatol. 2013;133(10):2444-52.

41. Hammett R, Shoemake C. In silico design and optimisation of Phytoalexin resveratrol polyphenolic analogs as inhibitors of histone acetyltransferase for the Management of Tumor Growth. Med Chem. 2018;8:242-9.

42. Seto E, Yoshida M. Erasers of histone acetylation: the histone deacetylase enzymes. Cold Spring Harb Perspect Biol. 2014:6(4):a018713-a.

43. Hadley M, Noonepalle S, Banik D, Villagra A. Functional analysis of HDACs in tumorigenesis. In: Brosh JRM, editor. Protein acetylation: methods and protocols. New York: Springer New York; 2019. p. 279-307.

44. Lee J, Ra SH. Cancer epigenetics: mechanisms and crosstalk of a HDAC inhibitor, Vorinostat. Chemotherapy. 2013;2(111):14934.

45. Reichert N, Choukrallah M-A, Matthias P. Multiple roles of class I HDACs in proliferation, differentiation, and development. Cell Mol Life Sci. 2012;69(13): 2173-87.

46. Bosch-Presegué L, Vaquero A. The dual role of sirtuins in cancer. Genes Cancer. 2011;2(6):648-62

47. Villagra A, Cheng F, Wang H-W, Suarez I, Glozak M, Maurin M, et al. The histone deacetylase HDAC11 regulates the expression of interleukin 10 and immune tolerance. Nat Immunol. 2009:10(1):92.

48. Eckschlager T, Plch J, Stiborova M, Hrabeta J. Histone deacetylase inhibitors as anticancer drugs. Int J Mol Sci. 2017:18(7):1414.

49. Makieva S, Giacomini E, Ottolina J, Sanchez A, Papaleo E, Viganò P. Inside the endometrial cell signaling subway: mind the gap (s). Int J Mol Sci. 2018; 19(9):2477. 
50. Mutter GL, Prat J. Pathology of the female reproductive tract. In: Mutter GL, Prat J, editors. . 3rd ed. Edinburgh: Churchill Livingstone Elsevier; 2014.

51. Marsh C, Schumacher K, Nothnick WB, Taylor RN, Monard M. Secretory phase and implantation. In: Menstrual cycle: IntechOpen. 2018. https://www. intechopen.com/books/menstrual-cycle/secretory-phase-and-implantation. Accessed 17 Sept 2019.

52. Wilson EW. In: Rennie PIC, editor. The menstrual cycle. London: LloydLuke; 1976.

53. Murdock TA, Veras EFT, Kurman RJ, Mazur MT. The Normal endometrium. In: Murdock TA, Veras EFT, Kurman RJ, Mazur MT, editors. Diagnosis of endometrial biopsies and Curettings: a practical approach. Cham: Springer International Publishing; 2019. p. 9-37.

54. Okada $H$, Tsuzuki T, Murata $H$. Decidualization of the human endometrium. Reprod Med Biol. 2018;17(3):220-7.

55. von Grothusen C, Lalitkumar S, Boggavarapu NR, Gemzell-Danielsson K, Lalitkumar PG. Recent advances in understanding endometrial receptivity: molecular basis and clinical applications. Am J Reprod immunol (New York, NY : 1989). 2014;72(2):148-57.

56. Gargett CE, Nguyen HPT, Ye L. Endometrial regeneration and endometrial stem/progenitor cells. Rev Endocr Metab Disord. 2012;13(4):235-51.

57. Gargett CE, Schwab KE, Deane JA. Endometrial stem/progenitor cells: the first 10 years. Hum Reprod Update. 2015;22(2):137-63.

58. Salamonsen LA. WOMEN IN REPRODUCTIVE SCIENCE: My WOMBan's life: understanding human endometrial function. Reproduction. 2019;158(6): F55-67.

59. Yakushiji N, Yokoyama H, Tamura K. Repatterning in amphibian limb regeneration: a model for study of genetic and epigenetic control of organ regeneration. Semin Cell Dev Biol. 2009;20(5):565-74.

60. Chung Y-L. Histone hyperacetylating agents for promoting wound healing and preventing scar formationGoogle Patents; 2015.

61. Figueira PGM, Abrão MS, Krikun G, Taylor HS. Stem cells in endometrium and their role in the pathogenesis of endometriosis. Ann N Y Acad Sci. 2011;1221(1):10-7.

62. Reyes M, Verfaillie C. Characterization of multipotent adult progenitor cells, a subpopulation of mesenchymal stem cells. Ann N Y Acad Sci. 2001;938: 231-5.

63. Wolf M, Kiesel L, Götte M. Stammzellen im endometrium. Gynäkologische Endokrinologie. 2009:7(3):185-9.

64. Gargett CE, Ye L. Endometrial reconstruction from stem cells. Fertil Steril. 2012;98(1):11-20.

65. Li X, Zhao X. Epigenetic regulation of mammalian stem cells. Stem Cells Dev. 2008;17(6):1043-52

66. Huang $B$, Li G, Jiang $X H$. Fate determination in mesenchymal stem cells: a perspective from histone-modifying enzymes. Stem Cell Res Ther. 2015:6(1):35.

67. Xu L, Xing Q, Huang T, Zhou J, Liu T, Cui Y, et al. HDAC1 silence promotes neuroprotective effects of human umbilical cord-derived mesenchymal stem cells in a mouse model of traumatic brain injury via PI3K/AKT pathway. Front Cell Neurosci. 2019:12:498.

68. Park S-Y, Phorl S, Jung S, Sovannarith K, Lee S-I, Noh S, et al. HDAC6 deficiency induces apoptosis in mesenchymal stem cells through p53 K120 acetylation. Biochem Biophys Res Commun. 2017;494(1):51-6.

69. Tan J, Lu J, Huang W, Dong Z, Kong C, Li L, et al. Genome-wide analysis of histone H3 Lysine9 modifications in human mesenchymal stem cell osteogenic differentiation. PLoS One. 2009:4(8):e6792.

70. Verdi J, Tan A, Shoae-Hassani A, Seifalian AM. Endometrial stem cells in regenerative medicine. J Biol Eng. 2014;8(1):20.

71. Ding D-C, Shyu W-C, Lin S-Z. Mesenchymal stem cells. Cell Transplant. 2011; 20(1):5-14.

72. Lu J, Kong X, Luo C, Li KK. Application of epigenome-modifying small molecules in induced pluripotent stem cells. Med Res Rev. 2013;33(4):790-822.

73. Mashayekhi P, Noruzinia M, Zeinali S, Khodaverdi S. Endometriotic mesenchymal stem cells epigenetic pathogenesis: deregulation of miR200b, miR-145, and let7b in a functional imbalanced epigenetic disease. Cell. 2019;21(2):179-85

74. Taghizadeh M, Noruzinia M. Lovastatin reduces Stemness via epigenetic reprograming of BMP2 and GATA2 in human endometrium and endometriosis. Cell. 2017;19(1):50-64.

75. Shukla V, Vaissière T, Herceg Z. Histone acetylation and chromatin signature in stem cell identity and cancer. Mutation Res/Fundamental Mol Mechanisms Mutagen. 2008;637(1):1-15.
76. Ferenczy A, Mutter G. The endometrial cycle. Glob Libr Women's Med. 2008. (ISSN: 1756-2228). https://doi.org/10.3843/GLOWM.10293. https:// www.glowm.com/section_view/heading/the-endometrial-cycle/item/292. Accessed 19 Sept 2019.

77. Gargett CE, Rogers PA. Human endometrial angiogenesis. ReproductionCambridge-. 2001;121(2):181-6.

78. Hayashi A, Horiuchi A, Kikuchi N, Hayashi T, Fuseya C, Suzuki A, et al. Typespecific roles of histone deacetylase (HDAC) overexpression in ovarian carcinoma: HDAC1 enhances cell proliferation and HDAC3 stimulates cell migration with downregulation of E-cadherin. Int J Cancer. 2010;127(6): 1332-46.

79. Ren J, Zhang J, Cai H, Li Y, Zhang Y, Zhang X, et al. HDAC as a therapeutic target for treatment of endometrial cancers. Curr Pharm Des. 2014;20(11): 1847-56.

80. Hrzenjak A, Moinfar F, Kremser M-L, Strohmeier B, Staber PB, Zatloukal K, et al. Valproate inhibition of histone deacetylase 2 affects differentiation and decreases proliferation of endometrial stromal sarcoma cells. Mol Cancer Ther. 2006;5(9):2203-10.

81. Fakhry H, Miyamoto $T$, Kashima H, Suzuki A, Ke H, Konishi I, et al. Immunohistochemical detection of histone deacetylases in endometrial carcinoma: involvement of histone deacetylase 2 in the proliferation of endometrial carcinoma cells. Hum Pathol. 2010;41(6):848-58.

82. Imesch $\mathrm{P}$, Fink D, Fedier $\mathrm{A}$. Romidepsin reduces histone deacetylase activity, induces acetylation of histones, inhibits proliferation, and activates apoptosis in immortalized epithelial endometriotic cells. Fertil Steril. 2010;94(7):2838-42.

83. Yi TZ, Li J, Han X, Guo J, Qu O, Guo L, et al. DNMT inhibitors and HDAC inhibitors regulate $\mathrm{E}$-cadherin and $\mathrm{BCl}-2$ expression in endometrial carcinoma in vitro and in vivo. Chemotherapy. 2012:58(1):19-29.

84. Ropero S, Esteller M. The role of histone deacetylases (HDACs) in human cancer. Mol Oncol. 2007;1(1):19-25.

85. Weichert W, Denkert C, Noske A, Darb-Esfahani S, Dietel M, Kalloger SE, et al Expression of class I histone deacetylases indicates poor prognosis in endometrioid subtypes of ovarian and endometrial carcinomas. Neoplasia. 2008:10(9):1021-7.

86. Jin G, Bausch D, Knightly T, Liu Z, Li Y, Liu B, et al. Histone deacetylase inhibitors enhance endothelial cell sprouting angiogenesis in vitro. Surgery. 2011;150(3):429-35.

87. Chen $Y$, Chang $Y$, Yao S. Role of angiogenesis in endometrial repair of patients with severe intrauterine adhesion. Int J Clin Exp Pathol. 2013;6(7): 1343-50.

88. Tamura I, Ohkawa Y, Sato T, Suyama M, Jozaki K, Okada M, et al. Genomewide analysis of histone modifications in human endometrial stromal cells. Mol Endocrinol. 2014:28(10):1656-69.

89. Gellersen B, Brosens IA, Brosens JJ. Decidualization of the human endometrium: mechanisms, functions, and clinical perspectives. Semin Reprod Med. 2007;25(6):445-53.

90. Van Den Brûle F, Berndt S, Simon N, Coulon C, Le Goarant J, Munaut C et al. Trophoblast invasion and placentation: molecular mechanisms and regulation. In: Immunology of gametes and embryo implantation. Basel, Karger. 2005;88:163-80.

91. Estella C, Herrer I, Atkinson SP, Quinonero A, Martinez S, Pellicer A, et al. Inhibition of histone deacetylase activity in human endometrial stromal cells promotes extracellular matrix remodelling and limits embryo invasion. PLoS One. 2012;7(1):e30508.

92. Bonnans $\mathrm{C}$, Chou J, Werb Z. Remodelling the extracellular matrix in development and disease. Nat Rev Mol Cell Biol. 2014;15(12):786-801.

93. Lala $\mathrm{P}$, Chakraborty C. Factors regulating trophoblast migration and invasiveness: possible derangements contributing to pre-eclampsia and fetal injury. Placenta. 2003;24(6):575-87.

94. Knöfler M. Critical growth factors and signalling pathways controlling human trophoblast invasion. Int J Dev Biol. 2010;54(2-3):269.

95. Sakai N, Maruyama T, Sakurai R, Masuda H, Yamamoto Y, Shimizu A, et al. Involvement of histone acetylation in ovarian steroid-induced Decidualization of human endometrial stromal cells. J Biol Chem. 2003:278(19):16675-82.

96. Kim H-J, Bae S-C. Histone deacetylase inhibitors: molecular mechanisms of action and clinical trials as anti-cancer drugs. Am J Transl Res. 2011;3(2):166-79.

97. Weichert W. HDAC expression and clinical prognosis in human malignancies. Cancer Lett. 2009;280(2):168-76.

98. Ahn MY, Lee J, Na YJ, Choi WS, Lee BM, Kang KW, et al. Mechanism of apicidin-induced cell cycle arrest and apoptosis in Ishikawa human endometrial cancer cells. Chem Biol Interact. 2009;179(2-3):169-77. 
99. Li L-H, Zhang P-R, Cai P-Y, Li Z-C. Histone deacetylase inhibitor, Romidepsin (FK228) inhibits endometrial cancer cell growth through augmentation of p53-p21 pathway. Biomed Pharmacother. 2016;82:161-6.

100. Bergadà L, Sorolla A, Yeramian A, Eritja N, Mirantes C, Matias-Guiu X, et al. Combination of Vorinostat and caspase-8 inhibition exhibits high antitumoral activity on endometrial cancer cells. Mol Oncol. 2013;7(4):763-75.

101. Yang S, Jia Y, Liu X, Winters C, Wang X, Zhang Y, et al. Systematic dissection of the mechanisms underlying progesterone receptor downregulation in endometrial cancer. Oncotarget. 2014;5(20):9783.

102. Yang S, Xiao X, Jia Y, Liu X, Zhang Y, Wang X, et al. Epigenetic modification restores functional PR expression in endometrial cancer cells. Curr Pharm Des. 2014;20(11):1874-80.

103. Fukuda T, Wada-Hiraike O, Oda K, Tanikawa M, Makii C, Inaba K, et al. Putative tumor suppression function of SIRT6 in endometrial cancer. FEBS Lett. 2015;589(17):2274-81.

104. Bartosch C, Monteiro-Reis S, Almeida-Rios D, Vieira R, Castro A, Moutinho M, et al. Assessing sirtuin expression in endometrial carcinoma and nonneoplastic endometrium. Oncotarget. 2016;7(2):1144-54.

105. Taguchi A, Wada-Hiraike O, Kawana K, Koga K, Yamashita A, Shirane A, et al. Resveratrol suppresses inflammatory responses in endometrial stromal cells derived from endometriosis: a possible role of the sirtuin 1 pathway. J Obstet Gynaecol Res. 2014;40(3):770-8.

106. Kawano Y, Nasu K, Li H, Tsuno A, Abe W, Takai N, et al. Application of the histone deacetylase inhibitors for the treatment of endometriosis: histone modifications as pathogenesis and novel therapeutic target. Hum Reprod. 2011;26(9):2486-98.

107. Xiaomeng X, Ming Z, Jiezhi M, Xiaoling F. Aberrant histone acetylation and methylation levels in woman with endometriosis. Arch Gynecol Obstet. 2013;287(3):487-94.

\section{Publisher's Note}

Springer Nature remains neutral with regard to jurisdictional claims in published maps and institutional affiliations.

\section{Ready to submit your research? Choose BMC and benefit from:}

- fast, convenient online submission

- thorough peer review by experienced researchers in your field

- rapid publication on acceptance

- support for research data, including large and complex data types

- gold Open Access which fosters wider collaboration and increased citations

- maximum visibility for your research: over $100 \mathrm{M}$ website views per year

At BMC, research is always in progress.

Learn more biomedcentral.com/submissions 